\section{Functional Molecular Imaging in Hepatology}

S. Keiding and M. Sørensen, eds.

Oak Park, IL: Bentham Science Publishers, 2012, 185 pages, $\$ 86$

Functional Molecular Imaging in Hepatology is a well-written, informative, and useful e-book by knowledgeable, dedicated authors. The first chapter gives a good overview of the hepatic vascular anatomy, describing the dual blood supply of the liver (the hepatic artery and portal vein), the special hepatic outflow system (the hepatic veins), and the essential role of substance exchange between blood and the highly permeable hepatic sinusoids in maintaining metabolic homeostasis. The key dynamic interplay of the hepatic vascular components for metabolism of endogenous and exogenous substances is essential for maintaining proper liver function.

Chapter 2 describes several methods that include intravascular tracers for dynamic PET measurement of total hepatic blood flow and perfusion of unit volume of liver tissue. Chapters 3 explains compartmental and microvascular models and describes research showing that, compared with compartmental models, the microvascular sinusoidal model of enzymatic elimination in vivo better explains the complex hepatic functions and in vivo effects of concentration gradients of substrates along capillaries. Chapter 4 discusses PET tracer use in measuring regional metabolism and in calculating the metabolic clearance of a mother substance based on clearance of its analog.

Chapter 5, on hepatic glucose and fatty acid metabolism, describes methodologic advances in liver metabolism and clinical findings seen by PET imaging in metabolic diseases. Chapter 6 , on biliary secretion, is a comprehensive, informative overview of the complex vital role played by the liver in preserving the balance of metabolic functions in the body. This chapter covers the detergent and detoxification role of bile; the protective role against colonic bacterial overgrowth; the role of biliary secretion, bile flow, and bile acids in normal digestion and disease; and cholesterol, triglyceride, and glucose and energy homeostasis. Ongoing medical research on quantification of liver metabolism and other medical challenges is also covered.

Chapter 7 introduces us to metabolomic analysis, a new technology for rapid, accurate, and precise evaluation of a large number of metabolic responses to liver disease. Besides being used in basic research, this technology has clinical application in the diagnosis and prognosis of liver disease and in determining the biochemical consequences of drug treatment. Chapters $8-11$ cover disease processes such as hepatocellular cancer, cholangiocarcinoma, liver metastases from colorectal cancer, and neuroendocrine tumors. The early diagnosis of hepatocellular cancer while the tumor is in a small, more curable stage using molecular imaging of specific metabolic pathways is beneficial compared with the traditional 4-phase contrast CT or dynamic contrastenhanced MR imaging. The authors discuss the improved diagnostic ability of ${ }^{18} \mathrm{~F}-\mathrm{FDG}$ PET in detecting recurrent cholangiocarcinoma and distant metastasis and the role of ${ }^{18} \mathrm{~F}-\mathrm{FDG}$ PET in colorectal cancer as the single best imaging modality for detecting intra- and extrahepatic metastasis or evaluating early therapy response. The introduction of newer PET tracers for neuroendocrine tumor work-up is covered. This chapter discusses the fact that ${ }^{18} \mathrm{~F}$-3,4-dihydroxyphenylalanine,

COPYRIGHT (C) 2014 by the Society of Nuclear Medicine and Molecular Imaging, Inc. a metabolism-based radiotracer, has limited use because of high production costs whereas the receptor-based compounds, ${ }^{68} \mathrm{Ga}$-DOTA peptides, are easier to use, are more economic, and localize better in well-differentiated neuroendocrine lesions than somatostatin receptor imaging agents. Also covered is the use of ${ }^{18} \mathrm{~F}$-FDG in undifferentiated neuroendocrine tumors with higher glucose metabolism or tumors with low expression of somatostatin receptors, such as medullary cancer of the thyroid.

Chapters 12-15 cover the neurologic effects of impaired hepatic function. Chapter 12 details the metabolic processes responsible for brain energy and ammonia metabolism. The effects of dysfunctional ammonia metabolism and toxic levels of ammonia in hepatic encephalopathy, brain energy metabolism, and amino acid metabolism are covered. Ongoing research tools to study these metabolic processes are discussed. Chapter 13 discusses the significant alterations in cerebral glucose utilization in cirrhosis with mild hepatic encephalopathy. ${ }^{18}$ F-FDG imaging shows decreased glucose utilization in the frontal cortex and anterior cingulate gyrus (which are involved in attention processes and motor performance), along with increased activity in the hippocampus, basal ganglia, and cerebellum.

Chapter 14 discusses cerebral ammonia metabolism in liver failure, including the finding of no change in cerebral ammonia kinetics or in the permeability-surface area product for ammonia across the bloodbrain barrier in cirrhotic patients with hepatic encephalopathy. The bulk of the evidence favors a correlation between metabolic trapping of ammonia and arterial blood ammonia concentration alone. Chapter 15 describes the relative merits of different MR techniques, including MR imaging and MR spectroscopy, and provides valuable insights into cerebral cell structure, water content, and metabolism in hepatic encephalopathy. The chapter also gives insight into neurologic dysfunction in liver diseases resulting from metal deposition (manganese, copper, and Wilson disease), hemochromatosis, and primary biliary cirrhosis and hepatitis $\mathrm{C}$.

As medical knowledge advances, its dissemination is taking novel approaches such as the e-book format, which will appeal to younger physicians who have grown up in the age of computers and the Internet. Some chapters, such as 2-4 and 12, are heavily biochemistry-oriented, requiring intense and dedicated concentration on the part of the reader to understand the many complex biochemical reactions covered. The clinically oriented chapters comprehensively cover useful information on hepatic function in health and disease and the toxic effects of ammonia and impaired hepatic function in hepatic encephalopathy. The effects of abnormal hepatic metabolism on functional molecular imaging are covered, as is future research using metabolic pathways and PET tracers. This textbook will be useful to hepatologists dealing with complex medical issues related to liver disease in their clinical practice. I also recommend the book to neurologists and to individuals involved in liver research.

\author{
Usha Joseph \\ University of Texas Medical School \\ 6431 Fannin, MSB 2.130 B \\ Houston, TX77030 \\ E-mail: usha.a.joseph@uth.tmc.edu
}

Published online Dec. 9, 2013

DOI: 10.2967/jnumed.113.133637 